\title{
Multiple plastic stents versus covered metal stent for treatment of anastomotic biliary strictures after liver transplantation : a prospective, randomized, multicenter trial
}

\section{Tal, Andrea Oliver}

2017-12

Tal , A O , Finkelmeier , F , Filmann , N , Kylänpää , L , Udd , M , Parzanese , I , Cantu , P , Dechene , A, Penndorf , V , Schnitzbauer , A, Friedrich-Rust, M, Zeuzem , S \& Albert , J G 2017 , ' Multiple plastic stents versus covered metal stent for treatment of anastomotic biliary strictures after liver transplantation : a prospective, randomized, multicenter trial ' , Gastrointestinal Endoscopy, vol. 86 , no. 6 , pp. 1038-1045 . https://doi.org/10.1016/j.gie.2017.03.009

http://hdl.handle.net/10138/298131

https://doi.org/10.1016/j.gie.2017.03.009

publishedVersion

Downloaded from Helda, University of Helsinki institutional repository.

This is an electronic reprint of the original article.

This reprint may differ from the original in pagination and typographic detail.

Please cite the original version. 


\title{
Multiple plastic stents versus covered metal stent for treatment of anastomotic biliary strictures after liver transplantation: a prospective, randomized, multicenter trial CME
}

\author{
Andrea Oliver Tal, MD, ${ }^{1}$ Fabian Finkelmeier, MD, ${ }^{1}$ Natalie Filmann, ${ }^{3}$ Leena Kylänpää, MD, PhD, ${ }^{5}$ \\ Marianne Udd, MD, PhD, ${ }^{5}$ Ilaria Parzanese, MD, ${ }^{4}$ Paolo Cantù, MD, PhD, ${ }^{4}$ Alexander Dechêne, MD, ${ }^{6}$ \\ Volker Penndorf, MD, ${ }^{6}$ Andreas Schnitzbauer, MD, Professor, ${ }^{2}$ Mireen Friedrich-Rust, MD, Professor, ${ }^{1}$ \\ Stefan Zeuzem, MD, Professor, ${ }^{1}$ Jörg G. Albert, MD, Professor ${ }^{7}$
}

Frankfurt, Germany; Milan, Italy; Helsinki, Finland; Essen, Germany; Stuttgart, Germany

\begin{abstract}
Background and Aims: Treatment of anastomotic biliary strictures (ABSs) after orthotopic liver transplantation by endoscopic insertion of multiple plastic stents (MPSs) is well established. The use of covered self-expandable metal stents (cSEMSs) for this indication is less investigated.
\end{abstract}

\begin{abstract}
Methods: In an open-label, multicenter, randomized trial, patients with confirmed ABSs were randomly assigned 1:1 to receive either an MPS or a cSEMS. The primary endpoint was the number of endoscopic interventions until ABS resolution. Secondary endpoints were frequency of adverse events, treatment success rates, and time to treatment success and recurrence of ABS during follow-up of at least 1 year.
\end{abstract}

Results: Fifty-eight patients were included between 2012 and 2015, and 48 patients completed follow-up. Patients receiving MPS $(n=24)$ underwent a median of 4 (range, 3-12) endoscopic retrograde cholangiography examinations, whereas those in the cSEMS group $(\mathrm{n}=24)$ underwent a median of 2 (range, 2-12) sessions until ABS resolution $(P<.001)$. A median of 8 (range, 2-32) stents was used until ABS resolution within the MPS group and 1 (range, 1-24) in the cSEMS group $(P<.0001)$. cSEMS migration occurred in 8 (33.3\%) patients. Treatment duration did not differ significantly. Initial treatment success rates were high with 23 (95.8\%) in the MPS group and $24(100 \%)$ for cSEMSs $(P=1)$. Five $(20.8 \%)$ patients in both groups showed stricture recurrence after a median follow-up of 500 days (range, 48-1317 days).

Conclusions: CSEMSs for treatment of ABSs needed less endoscopic interventions to achieve similar efficacy as MPS and might become a new treatment standard. However, the optimal duration of cSEMS therapy and costefficacy have to be evaluated. (Clinical trial registration number: NCT01393067.) (Gastrointest Endosc 2017;86:1038-45.)

\section{INTRODUCTION}

Anastomotic biliary stricture (ABS) is a major cause of morbidity after orthotopic liver transplantation (OLT)

\begin{abstract}
Abbreviations: $A B S$, anastomotic biliary stricture; $C B D$, common bile duct; cSEMS, covered self-expandable metal stent; ERC, endoscopic retrograde cholangiography; MPSs, multiple plastic stents; OLT, orthotopic liver transplantation.
\end{abstract}

DISCLOSURE: All authors disclosed no financial relationships relevant to this publication.

See CME section; p. 1151.

Copyright $\odot 2017$ by the American Society for Gastrointestinal Endoscopy 0016-5107/\$36.00

http://dx.doi.org/10.1016/j.gie.2017.03.009

Received October 20, 2016. Accepted March 5, 2017. with a reported incidence of $8 \%$ to $20 \% .{ }^{1-3}$ Nonsurgical management of ABS has become the first-line treatment strategy. ${ }^{4}$ This therapy consists of endoscopic or percutaneous stent placement, hydrostatic balloon

Current affiliations: Department of Internal Medicine I, Frankfurt University Hospital, Frankfurt, Germany (1), Department of General and Visceral Surgery, Frankfurt University Hospital, Frankfurt, Germany (2), Institute of Biostatistics and Mathematical Modeling, Frankfurt University Hospital, Frankfurt, Germany (3), Department of Gastroenterology, IRCCS Cà Granda, Ospedale Policlinico, Milan, Italy (4), Department of Gastrointestinal and General Surgery, Helsinki University Central Hospital, Helsinki, Finland (5), Department of Gastroenterology and Hepatology, Universitätsklinikum Essen, Essen, Germany (6), Department of Gastroenterology, Hepatology and Endocrinology, Robert-Bosch Hospital, Stuttgart, Germany (7).

Reprint requests: Dr. Andrea Tal, MD, Theodor-Stern-Kai 7, Medizinische Klinik I, Universitätsklinik Frankfurt am Main, 60590 Frankfurt, Germany. 
dilation, or a combination of both techniques, which can be applied via endoscopic retrograde cholangiography (ERC) or percutaneous transhepatic cholangiography technique. ${ }^{5}$ Primary success rates of up to $90 \%$ are reported but strictures recur in approximately 20\% of patients. ${ }^{6}$ Even after ABS recurrence, endoscopic retreatment is feasible with $90 \%$ to $100 \%$ resolution rates. ${ }^{3,7,8}$ In most centers, placement of multiple plastic stents (MPSs) with an increasing number of stents at each stent exchange is used. Patients with ABSs typically need 4 to 5 interventions by ERCP until the stricture is dilated sufficiently. $^{9-12}$

However, from the patients' perspective, repetitive ERCP sessions for plastic stent exchange have a negative effect on compliance and acceptance of this treatment approach. With covered self-expandable metal stents (cSEMSs), this limitation can be challenged: Efficacy of cSEMSs in the treatment of benign strictures has been shown to be noninferior to MPSs. ${ }^{12}$ Furthermore, implantation of a single cSEMS has a diameter equivalent to several $10 \mathrm{~F}$ plastic stents but offers longer patency ${ }^{13}$; therefore, stent exchanges are required less frequently as the stent indwelling time of cSEMSs is usually much longer than for plastic stents. Only a very limited number of randomized trials comparing the efficiency of MPSs versus cSEMSs for the treatment of ABSs are available. ${ }^{15-17}$

We conducted an open-label, multicenter, randomized trial to compare MPSs and cSEMSs with regard to the number of endoscopic sessions, adverse events, and success rates in the treatment of ABSs.

\section{PATIENTS AND METHODS}

\section{Patient selection}

The study was conducted as a prospective, open-label, multicenter trial (clinical trials registration number: NCT01393067) in 4 European liver transplantation centers after approval of their local ethics committees (Frankfurt University Hospital, Germany; Essen University Hospital, Germany; Department of Gastroenterology, IRCCS Cà Grande, Ospedale Policlinico, Milan, Italy; Department of Gastrointestinal and General Surgery, Helsinki University Central Hospital, Helsinki, Finland). All patients who, between 2012 and 2015, underwent deceased donor OLT for end-stage liver disease or acute liver failure were eligible for this study if ABS was suspected or confirmed by ERC. All patients included in this study did not participate in other studies. Patients with nonanastomotic strictures, including ischemic-type biliary lesions or biliodigestive anastomosis, were excluded. ABS was clinically suspected if signs of cholangitis, pruritus, or progressive jaundice were observed by clinical examination and/or abdominal ultrasonography revealed cholestasis. After obtaining informed consent and confirmation of ABS via ERC, patients were randomized 1:1 to receive either MPS or cSEMSs. ABS was defined as a stricture occurring at or directly adjacent to the choledocho-choledochal anastomosis. Patients were not included in the study if either MPSs or cSEMSs were judged technically not feasible or reasonable by the investigator.

\section{Endoscopic treatment}

For ERCP therapeutic duodenoscopes (eg, TJF160 VR or TJF Q180 VR; Olympus Corporation, Tokyo, Japan) were used by endoscopists with experience of $>1000$ ERCs.

The stricture grade was determined by a modification of a former published grading system, which was assessed by fluoroscopic imaging (stricture grade A, 99\%-100\% of luminal diameter; B, 90\%-99\%; C, 50\%-90\%; D, <50\%; E, $0 \%){ }^{3}$ After confirmation and grading of ABSs, endoscopic sphincterotomy was performed in all patients. Whether to perform hydrostatic balloon dilatation before stent placement (eg, 6 or $8 \mathrm{~mm}$ MaxForce balloon catheter; Boston Scientific, Ratingen, Germany) was at the discretion of the investigator. Participants were treatment naive with regard to ABSs, except for previous emergency treatment with single plastic stents in cases of acute cholangitis.

In the MPS group, the investigator inserted as many plastic endoprostheses as possible choosing the optimal diameter at his discretion (eg, 7F, 10F, 11.5F). Every 6 to 12 weeks, stent exchange ERC sessions were scheduled, and the number and diameter of the MPSs were increased as considered appropriate by the investigators. Within this trial, 4 different plastic stent types were used: straight plastic stents with 2 flaps, plastic stents with a duodenal bend and 2 flaps, plastic stents with a central bend and 2 flaps, or double pigtail stents.

Patients randomized to the cSEMS group received a fully covered stent with a diameter of $10 \mathrm{~mm}$. Three different cSEMSs were used within this trial; all were fully covered and without antimigration flaps. For retrieval, 2 stent types had a small retrieval flap and 1 cSEMS had a big lasso. Two cSEMS were placed across the papilla and 1 cSEMS type was placed completely inside the common bile duct (CBD). After 4 to 6 months, an ERC session was scheduled for cSEMS removal. In both groups, treatment was defined to be successful if no relevant ABS was left at the stent removal/exchange session. Patients regularly visited the hospitals' outpatient clinic, and clinical examinations including blood sampling and sonography were performed. Endoscopic therapy was determined successful in cases of radiomorphologic resolution of the stricture with persisting narrowing of the anastomosis of less than $30 \%$ of the diameter of the CBD.

Investigators were allowed to perform ERCP earlier than per protocol if there was clinical suspicion of stent occlusion or migration. In cases of any adverse events, eg, stent migration, stent dysfunction, or hemobilia, a crossover to the other treatment arm was advised. During a follow-up period of 1 year, patients presented routinely every 2 to 


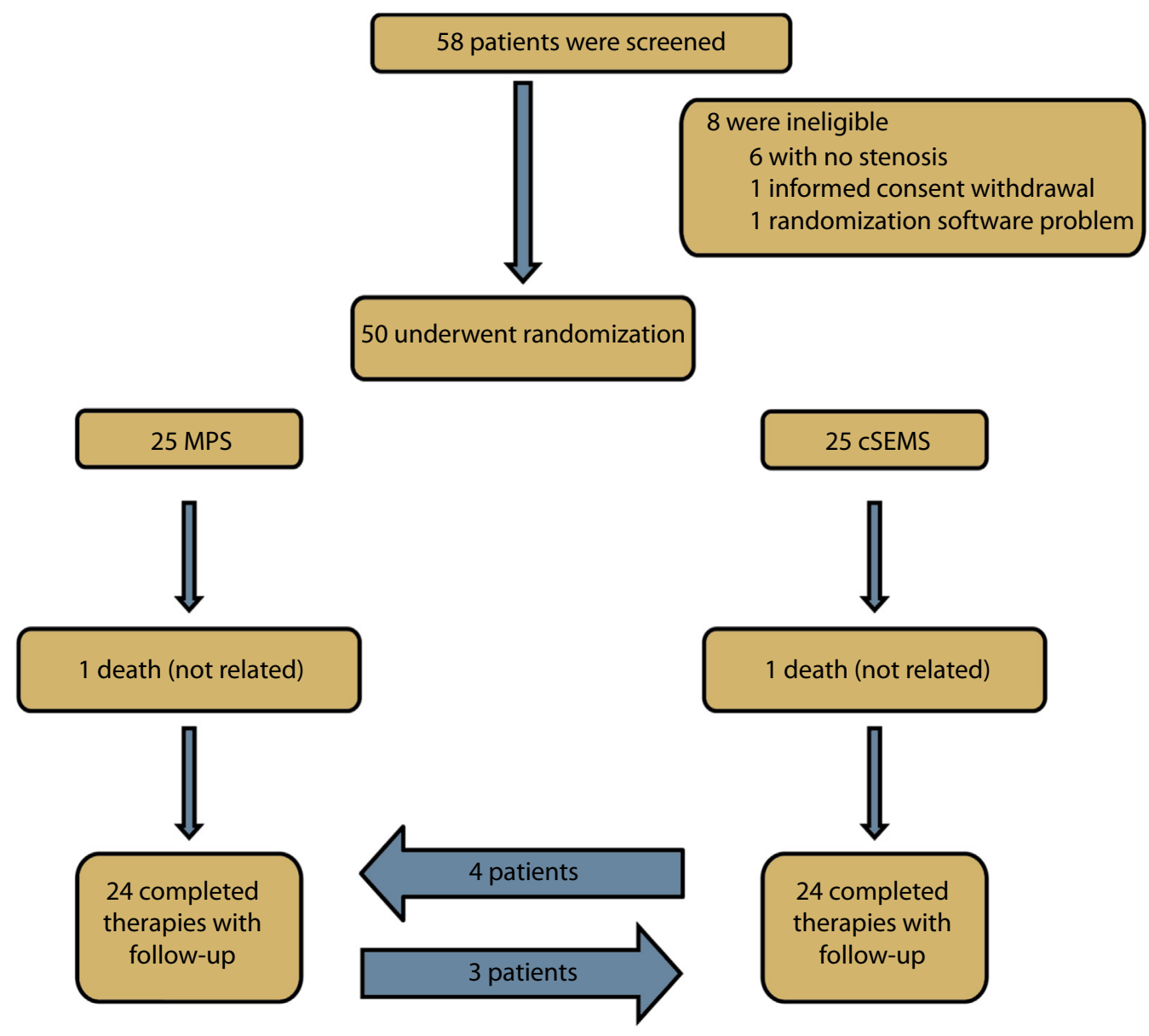

Figure 1. Patient flowchart for this study. cSEMS, Covered self-expandable metal stent; MPS, multiple plastic stents.

3 months in our interdisciplinary transplantation clinic where abdominal ultrasound and liver function tests were performed to evaluate whether a relevant ABS recurrence occurred.

The primary endpoint was the number of ERC sessions until resolution of ABS. Secondary endpoints were frequency of adverse events, treatment success rates of the respective stent regimens, time to treatment success, and recurrence of $\mathrm{ABS}$ during follow-up.

\section{Statistical analysis}

Fax randomization was provided by the Institute of Biostatistics and Mathematical Modeling, Goethe University, Frankfurt, Germany. Center-stratified block randomization was conducted using the R package "blockrand" (R Development Core Team [2010] [R Foundation for Statistical Computing, Vienna, Austria]). Patient-related data were pseudonymized in case record forms and collected at the study center in Frankfurt for analysis in perprotocol and intention-to-treat fashion. The primary endpoint of this study was the number of ERC sessions. We assumed that the number of interventions was described by random variables that follow a Poisson distribution with parameters $\lambda 1$ und $\lambda 2$. The mean number of interventions was thereby compared using a Poisson test. The test was 2 -sided with a level of significance of $\alpha=$ $5 \%$. Data from the literature were used to determine $\lambda 1$ and $\lambda 2$. The estimated number of investigations for MPS treatment was 5 , and the estimated number of investigations for cSEMS was 2 (implantation and explantation). ${ }^{18}$ With 25 patients in each therapy group, a 2-sided Poisson test can detect a difference between the 2 study groups with a level of significance of $\alpha=5 \%$ and a power of $1-\beta$ of at least $80 \%$.

\section{Data analysis}

Continuous variables are shown as the median and range, and categorical variables are reported as frequencies and percentages. Differences among different patient cohorts were determined using the Fisher exact test or Fisher-Freeman-Halton exact test for categorical variables. For quantitative variables, we used the Mann-Whitney U test or Kruskal-Wallis test. All tests were 2-sided, and $P$ values $<.05$ were considered to be significant; if necessary 


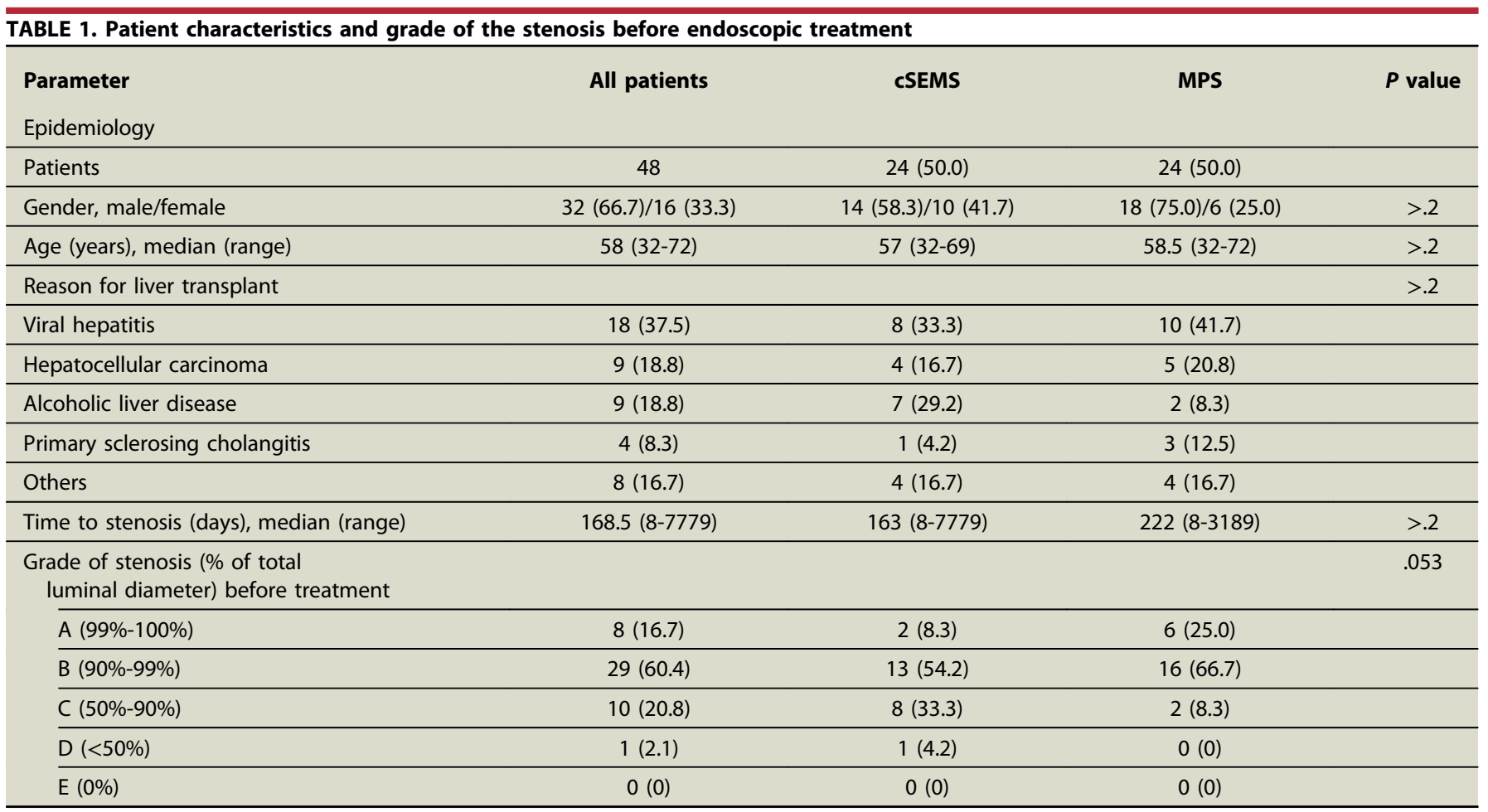

Values are number (\%) unless indicated otherwise.

cSEMS, Covered self-expandable metal stent; MPS, multiple plastic stents.

$P$ value corrections were administered. Statistical analyses were performed with SPSS (version 22.0, IBM, New York, NY) and GraphPad Prism (version 5, GraphPad, La Jolla, Calif). All authors reviewed and approved the final version of this manuscript.

\section{RESULTS}

Fifty-eight patients were enrolled between July 2012 and April 2015; 48 patients completed follow-up (MPS group, $\mathrm{n}=24$; cSEMS group, $\mathrm{n}=24$ ) (Fig. 1). Of 58 eligible patients, 6 had no relevant stenosis verified by fluoroscopy within ERC. One patient was excluded from the study because of a technical failure at randomization and 1 patient withdrew consent after enrollment. Two patients died during follow-up from non-study-related morbidity. One patient (cSEMS group) died after multiorgan failure caused by urosepsis. The other patient (MPS group) died at home supposedly from cardiac arrest.

The median age of the patients treated with MPS was 58.5 years (range, 32-72 years) compared with 57 years (range, 32-69 years) in the cSEMS treatment group $(P=.718)$. Gender distribution was similar in both groups; 6 females were treated with MPS and 10 females received cSEMS treatment $(P=.359)$. The most frequent indication for OLT was liver cirrhosis caused by viral hepatitis (MPS group, $\mathrm{n}=10,41.7 \%$; cSEMS group, $\mathrm{n}=8$,
33.3\%) followed by hepatocellular carcinoma in cirrhotic patients (MPS, $\mathrm{n}=5,20.8 \%$; cSEMS, $\mathrm{n}=4,16.7 \%$ ) (Table 1). Time between OLT and endoscopic treatment was a median of 222 days (range, 8-3189 days) in patients treated with MPS and 163 days (range, 8-7779 days) in the cSEMS group $(P=.861)$. ABSs were filiform (A) in 8 (16.7\%), high-grade (B) in 29 (60.4\%), moderate $(C)(\mathrm{n}=10,20.8 \%)$, or low-grade (D) $(\mathrm{n}=$ $1,2.1 \%)$ before endoscopic treatment. ABS grade did not significantly differ between both treatment arms $(P=.053)$. Balloon dilatation of ABS was performed in 14 (58.3\%) and 10 (41.6\%) patients before the first stent placement in the MPS versus cSEMS treatment arm $(P=.387)$.

In the cSEMS group, we observed hepatofugal stent migration in 8 patients (33.33\%). In 4 (50\%) of these cases, ABS was already resolved and no further endoscopic treatment was necessary at the time of follow-up. One patient (12.5\%), however, later developed recurrent symptomatic $\mathrm{ABS}$ and had to undergo repeat treatment. In the remaining 4 (50\%) patients, ABS was still detectable at follow-up ERC and crossover to treatment by MPS insertion was initiated. In 5 of the 8 patients (62.5\%) with spontaneous cSEMS migration, the cSEMS was excreted via naturalis on the day of ERC; in 3 cases $(37.5 \%)$, the dislocations were not observed by the patient or the investigator.

In 1 patient in the MPS group (4.1\%), severe hemobilia was observed during stent exchange, and therefore a 

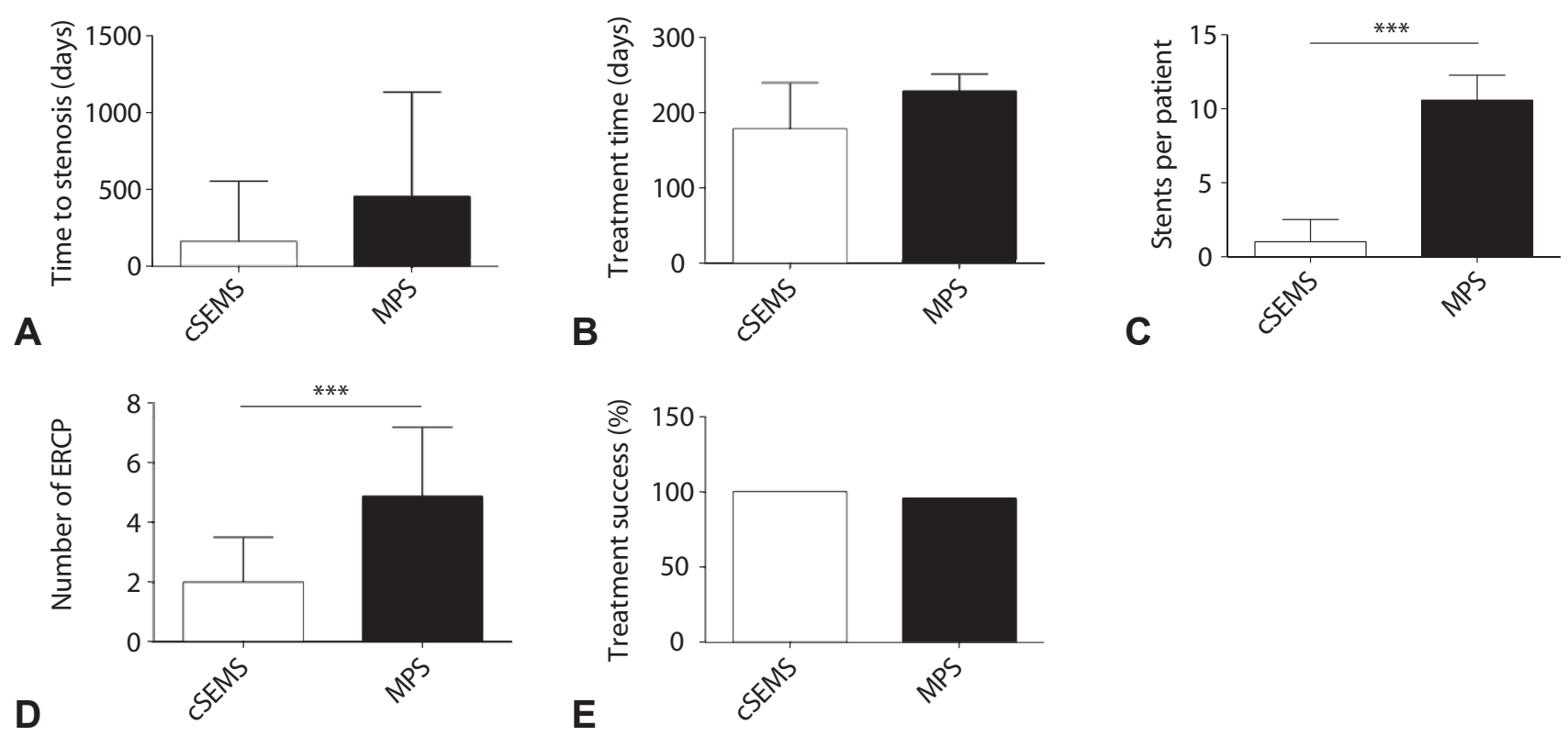

Figure 2. Intention-to-treat analysis. A, Time until development of ABS in days (median + interquartile range, $P=.861$ ), $\mathbf{B}$, Median endoscopic treatment time (median + interquartile range, $P=.458$ ). $\mathbf{C}$, Total number of stents inserted per patient (median + interquartile range, $P<.0001$ ). $\mathbf{D}$, Median number of ERCs performed (median + interquartile range, $P<.0001)$. E, Percentage treatment success $(P>.2)$. cSEMS, Covered self-expandable metal stent; MPSs, multiple plastic stents.

cSEMS was inserted to stop bleeding. In 1 patient (4.1\%) who was initially treated with MPS, treatment was discontinued because of spontaneous development of a bilioduodenal fistula no longer requiring biliary drainage. In 3 patients (12.5\%), MPS treatment was switched to cSEMS treatment, 1 because of severe bleeding, 1 insisted on receiving a cSEMS because of a very long driving distance to the hospital, and 1 patient for unknown reasons.

\section{Intention-to-treat analysis}

Patients treated with MPS underwent a median of 4 (range, 3-12) ERCs, whereas patients in the cSEMS group underwent a median of 2 (range, 2-12) sessions until ABS resolution when including the crossover treatments $(P<.001)$ (Fig. 2, Table 2). The number of implanted stents per patient until end of treatment was 8 (range, 232 ) in the MPS group and 1 (range, 1-24) in the cSEMS group $(P<.0001)$, including patients who were converted from cSEMS to MPS treatment and vice versa. Investigators used a median of 2.5 (range, 1-5) stents for upsizing at every programmed stent exchange in the MPS group compared with only 1 stent (range, 1-4) in the cSEMS group $(P<.0001)$. Median time until the end of treatment was 229.5 days (range, 59-490 days) in the MPS group and 178.5 days (range, 65-551 days) in the CSEMS group $(P=.458)$. Both treatment arms achieved high initial treatment success rates of $95.8 \%(\mathrm{n}=23)$ for patients treated with MPS and 100\% ( $\mathrm{n}=24)$ for those treated with cSEMSs $(P=1)$. There was no difference found in the incidence of ABS recurrence between the 2 different treatment groups with 5 relapses $(20.8 \%)(P=1)$ in each group after a median follow-up of 500 days (range, 48-1317 days) after endoscopic treatment.

\section{Per-protocol analysis}

Twenty of 24 patients (83.3\%) who were initially allocated to treatment with MPS (Fig. 3) and 18 of 24 patients $(75 \%)$ who were treated with cSEMS completed endoscopic treatment of ABS $(P=1)$ (Table 3). ABS grade before treatment did not differ between the groups. Gender distribution did not significantly differ with 5 females in the MPS group and 7 females in the cSEMS group $(P=.569)$. The median age of patients receiving MPSs was 58.5 years (range, $32-72$ years) compared with 58.5 years (range, 37-69 years) for patients treated with CSEMS $(P=.675)$. A median of 239.5 (range, 59-490) and 177.5 (range, 65-249) treatment days was required for ABS resolution for patients in the MPS versus cSEMS groups, respectively $(P=.015)$. In the MPS group, a median of 4 (range, 3 12) versus 2 (range, 2-2) endoscopic interventions in the cSEMS arm were performed $(P<.0001)$ and a total of 9 (range, 2-32) versus 1 stents were inserted $(P<$ $.0001)$. ABS recurrence was observed in $20 \%$ versus $22.2 \%$ of patients in the MPS versus cSEMS groups, respectively $(P=1)$. During a median follow-up of 508 days (range, $65-1183$ days) $(P=.093)$ for patients treated with MPS and 399.5 days (range, 189-1048 days) for those treated with cSEMSs (Fig. 4), the recurrence rate was not 


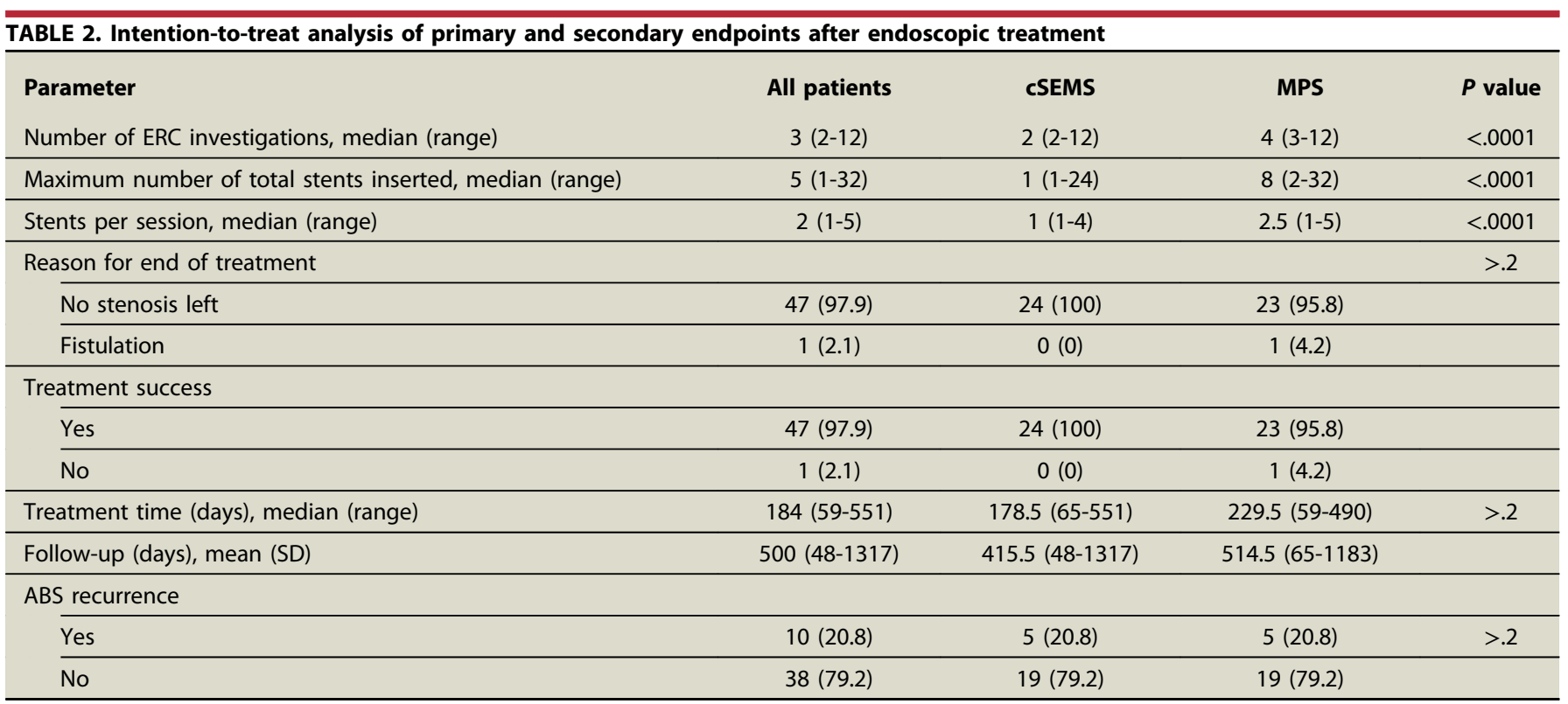

Values are number (\%) unless indicated otherwise.

CSEMS, Covered self-expandable metal stent; MPSs, multiple plastic stents; $E R C$, endoscopic retrograde cholangiography; $S D$, standard deviation; $A B S$, anastomotic biliary stricture.
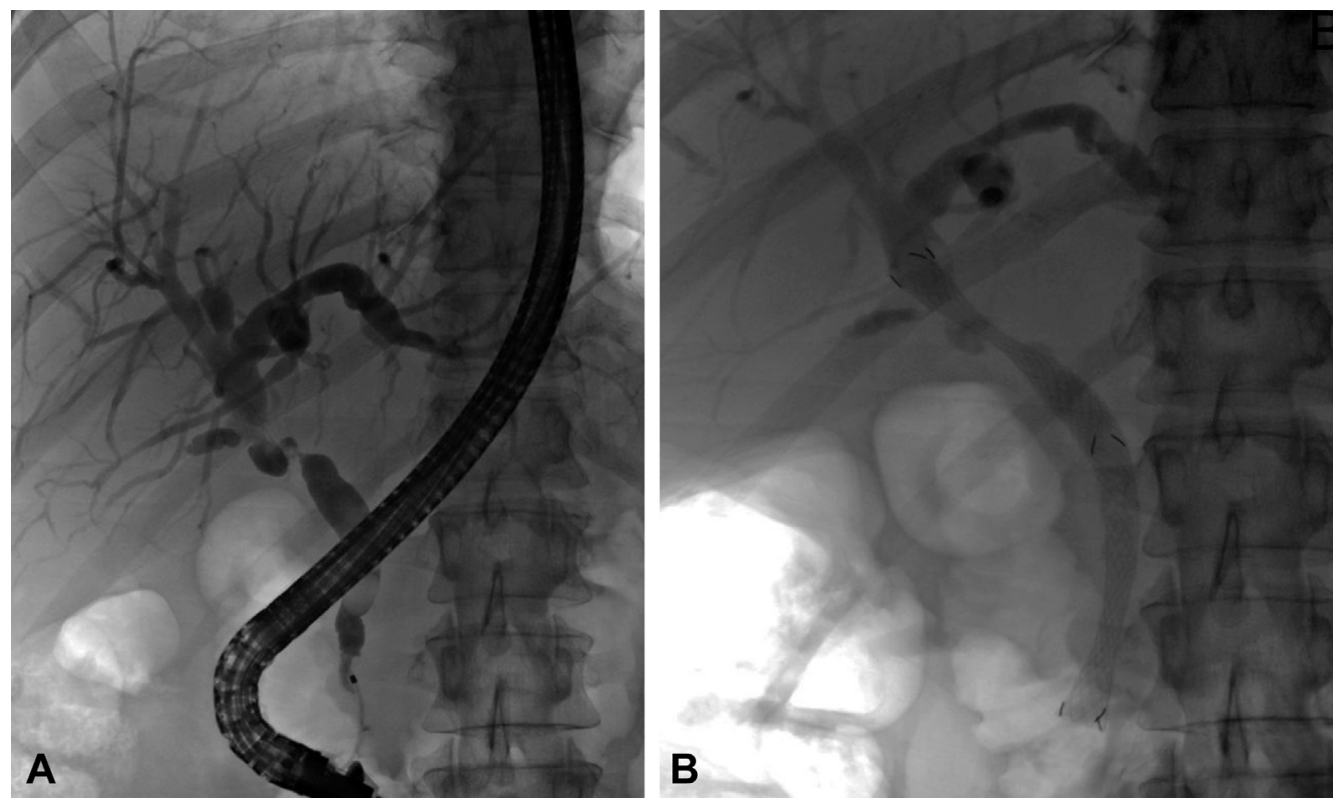

Figure 3. cSEMS treatment. A, Fluoroscopic visualization of the stenosis. B, Stenosis after cSEMS insertion. cSEMS, Covered self-expandable metal stent.

significantly different at $20 \%(\mathrm{n}=4)$ versus $22.2 \%$ $(\mathrm{n}=4)(P=1)$.

\section{DISCUSSION}

Endoscopic therapy is the standard treatment modality for ABS after OLT, and insertion of multiple plastic stents is widely practiced. We previously published that this approach is highly effective with a success rate of about
90\%, and that ABS recurrence after initial stricture resolution can again be successfully treated endoscopically. ${ }^{3}$ However, as several ERC sessions for stent exchange are required until stricture resolution, a more efficient and, for the patient, more comfortable alternative may increase acceptance of this therapy. Recently, several investigators reported cSEMSs to be safe and efficient for treatment of benign as well as malignant CBD strictures, ie, tumor obstruction, non-neoplastic strictures, and particularly ABSs. ${ }^{13,19,20}$ 


\begin{tabular}{|c|c|c|c|c|}
\hline \multicolumn{5}{|l|}{ Epidemiology } \\
\hline Patients & 38 & 18 & 20 & \\
\hline Gender, male/female & $26(68.4 \%) / 12(31.6 \%)$ & $11(61.1) / 7(38.9)$ & $15(75) / 5(25)$ & $>.2$ \\
\hline Age (years), median (range) & $58.5(32-72)$ & $58.5(37-69)$ & $58.5(32-72)$ & $>.2$ \\
\hline Grade of stenosis before treatment & & & & .095 \\
\hline A (99\%-100\%) & $6(15.8)$ & $1(5.6)$ & $5(25)$ & \\
\hline B (90\%-99\%) & $23(60.5)$ & $10(55.6)$ & $13(65)$ & \\
\hline C (50\%-90\%) & $8(21.1)$ & $6(33.3)$ & $2(10)$ & \\
\hline Maximum number of total stents inserted, median (range) & $3.5(1-32)$ & $1(1-1)$ & $9(2-32)$ & $<.001$ \\
\hline Stents per session, mean (range) & $1.5(1-5)$ & 1 & $3(1-5)$ & $<.001$ \\
\hline Primary treatment success & & & & $>.2$ \\
\hline Yes & $38(100)$ & $18(100)$ & $20(100)$ & \\
\hline No & $0(0)$ & $0(0)$ & $0(0)$ & \\
\hline Treatment time (days), median (range) & $180.5(59-490)$ & $177.5(65-249)$ & $239.5(59-490)$ & .015 \\
\hline Follow-up (days), median (range) & $495.5(65-1183)$ & $399.5(189-1048)$ & $508(65-1183)$ & .093 \\
\hline Recurrence & & & & $>.2$ \\
\hline Yes & $8(21.1)$ & $4(22.2)$ & $4(20)$ & \\
\hline
\end{tabular}

Values are number (\%) unless indicated otherwise.

CSEMS, Covered self-expandable metal stent; MPSs, multiple plastic stents; OLT, orthotopic liver transplantation; ERC, endoscopic retrograde cholangiography.
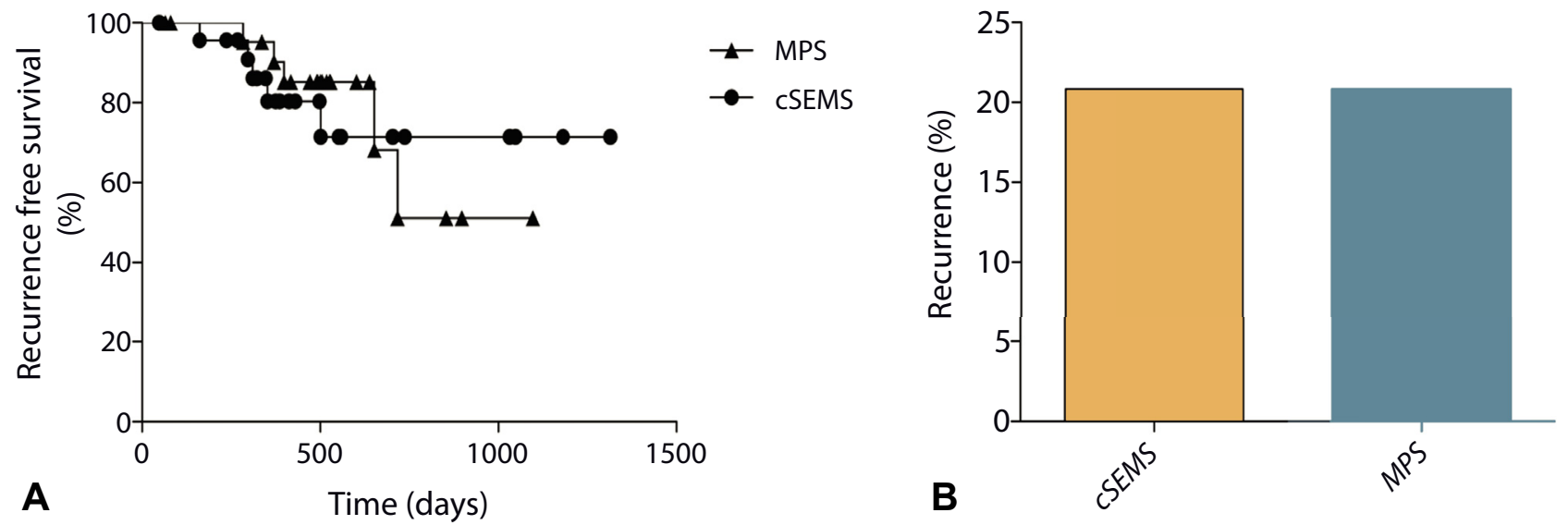

Figure 4. Recurrence-free survival. A, Kaplan-Meier curve of ABS recurrence (log-rank test, $P=.9$ ). Not achieved in $50 \%$; therefore, no median survival rates could be calculated. B, Percentage ABS recurrence (median + interquartile range, $P>.2$ ). cSEMS, Covered self-expandable metal stent; $M P S s$, multiple plastic stents.

In this prospective randomized study, we observed that cSEMS treatment of ABSs offers a reduction in treatment time and number of interventions compared with the current treatment standard, ie, MPS insertion. Both the intention-to-treat as well as the per-protocol analyses demonstrated fewer interventions and stents per patient for cSEMSs. For MPSs, a median of 4 ERC examinations was needed compared with 2 sessions until ABS resolution in the cSEMS group $(P<.001)$ in the intention-to-treat analysis. A median of 8 stents was used until the end of treatment in the MPS group and 1 in the cSEMS group. This is in line with a recently published trial where the 
mean number of ERCPs to achieve stricture resolution was lower for cSEMSs (2.14) versus plastic stents (3.24) (mean difference, 1.10 ; 95\% confidence interval, 0.74 to $1.46 ; P<$ .001 ) in this randomized trial. ${ }^{14,15}$

ABS recurrence rates were equal in both treatment groups after 1 year of follow-up. This may indicate that MPSs and cSEMSs provide similar radial expansion force, and clinical results may be equal for similar treatment duration. However, long-time ABS recurrence of about 20\% after initial endoscopic treatment success indicates that treatment duration should be long enough irrespective of the stent type, MPS or cSEMS. ${ }^{3}$ Comparative trials have not yet addressed the most appropriate treatment duration, however, expert opinion advises treatment at at least 4 to 6 months. ${ }^{13}$

In this study, 8 of 24 (33\%) cSEMSs spontaneously migrated. Four of these patients did not need any further treatment because there was no stricture remaining. The other 4 patients continued treatment with MPSs. We used 3 different types of cSEMSs within this trial. Some were placed across the papilla, and 1 cSEMS type was placed completely inside the CBD. We could see a tendency toward less migration for cSEMSs when placed completely within the CBD but because of low patient numbers, no statistically valid calculations or statements can be made. Considering the tendency toward less stent migration with cSEMSs that are placed completely within the bile duct, our findings are in agreement with the results of Kaffes et al. ${ }^{15}$ Therefore, new antimigration measures for cSEMSs are needed as well as randomized trials comparing different types of cSEMSs. An ideal cSEMS would have a high radial expansion force without spontaneously migrating after or even before stricture resolution. Only 1 patient with a cSEMS migration developed recurrent ABS. ${ }^{4,13}$

Some limitations have to be taken into account. The study is quite small by number; however, it is multicenter and prospective, thereby reflecting daily practice.

Although cSEMSs seem to have advantages over MPSs, cSEMSs are still quite expensive, and a thorough cost analysis was not carried out. However, the reduced number of treatments should at least counterbalance the increased costs of the stent. Furthermore, there are several different kind of stents available, and we did not limit use to one single kind of stent. Therefore, it is possible that some cSEMSs are superior to others, which should be investigated in the future.

In summary, for cSEMS treatment of ABS, fewer treatment sessions are necessary. Obviously, cSEMSs and MPSs are comparably safe and efficient and achieve a similar outcome. Our study adds evidence to the growing amount of data showing that cSEMS treatment for ABSs is a good alternative to MPSs. However, improved stent models should decrease migration rates of cSEMSs. The optimal duration of cSEMS therapy and cost-efficacy analysis of cSEMSs versus MPSs are subject to future studies.

\section{REFERENCES}

1. Stratta RJ, Wood RP, Langnas AN, et al. Diagnosis and treatment of biliary tract complications after orthotopic liver transplantation. Surgery 1989;106:675-83.

2. Suárez F, Otero A, Solla M, et al. Biliary complications after liver transplantation from Maastricht category-2 non-heart-beating donors. Transplantation 2008;85:9-14.

3. Albert JG, Filmann N, Elsner J, et al. Long-term follow-up of endoscopic therapy for stenosis of the biliobiliary anastomosis associated with orthotopic liver transplantation. Liver Transpl 2013;19: 586-93.

4. Williams ED, Draganov PV. Endoscopic management of biliary strictures after liver transplantation. World J Gastroenterol 2009;15: 3725-33.

5. Kulaksiz H, Weiss KH, Gotthardt D, et al. Is stenting necessary after balloon dilation of post-transplantation biliary strictures? Results of a prospective comparative study. Endoscopy 2008;40:746-51.

6. Tabibian JH, Asham EH, Han S, et al. Endoscopic treatment of postorthotopic liver transplantation anastomotic biliary strictures with maximal stent therapy (with video). Gastrointest Endosc 2010;71:505-12.

7. Tringali A, Barbaro F, Pizzicannella M, et al. Endoscopic management with multiple plastic stents of anastomotic biliary stricture following liver transplantation: long-term results. Endoscopy 2016;48:546-51.

8. Hsieh T-H, Mekeel KL, Crowell MD, et al. Endoscopic treatment of anastomotic biliary strictures after living donor liver transplantation: outcomes after maximal stent therapy. Gastrointest Endosc 2013;77: 47-54.

9. Graziadei IW, Schwaighofer H, Koch R, et al. Long-term outcome of endoscopic treatment of biliary strictures after liver transplantation. Liver Transpl 2006;12:718-25.

10. Verdonk RC, Buis $\mathrm{Cl}$, Porte RJ, Haagsma EB. Biliary complications after liver transplantation: a review. Scand J Gastroenterol Suppl 2006;(243):89-101.

11. Pasha SF, Harrison ME, Das A, et al. Endoscopic treatment of anastomotic biliary strictures after deceased donor liver transplantation: outcomes after maximal stent therapy. Gastrointest Endosc 2007;66:44-51.

12. Morelli J, Mulcahy HE, Willner IR, Cunningham JT, Draganov P. Longterm outcomes for patients with post-liver transplant anastomotic biliary strictures treated by endoscopic stent placement. Gastrointest Endosc 2003;58:374-9.

13. Devière J, Nageshwar Reddy D, Püspök A, et al. Successful management of benign biliary strictures with fully covered self-expanding metal stents. Gastroenterology 2014;147:385-95; quiz e15.

14. Coté GA, Slivka A, Tarnasky P, et al. Effect of covered metallic stents compared with plastic stents on benign biliary stricture resolution: a randomized clinical trial. JAMA 2016;315:1250-7.

15. Kaffes A, Griffin S, Vaughan R, et al. A randomized trial of a fully covered self-expandable metallic stent versus plastic stents in anastomotic biliary strictures after liver transplantation. Therap Adv Gastroenterol [Internet] 2014;7:64-71.

16. Tarantino I, Traina M, Mocciaro F, et al. Fully covered metallic stents in biliary stenosis after orthotopic liver transplantation. Endoscopy 2012;44:246-50.

17. Kao D, Zepeda-Gomez S, Tandon P, Bain VG. Managing the post-liver transplantation anastomotic biliary stricture: multiple plastic versus metal stents: a systematic review. Gastrointest Endosc 2013;77:679-91.

18. Friedman S, Odze RD, Farraye FA. Management of neoplastic polyps in inflammatory bowel disease. Inflamm Bowel Dis 2003;9:260-6.

19. Walter D, Sarrazin C, Trojan J, et al. No distal migration in unfixed versus fixed cell structure covered self-expanding metal stents for treatment of benign biliary disease. Dig Dis Sci 2015;60: 2495-501.

20. Kahaleh M, Brijbassie A, Sethi A, et al. Multicenter trial evaluating the use of covered self-expanding metal stents in benign biliary strictures: time to revisit our therapeutic options? J Clin Gastroenterol 2013;47: 695-9. 